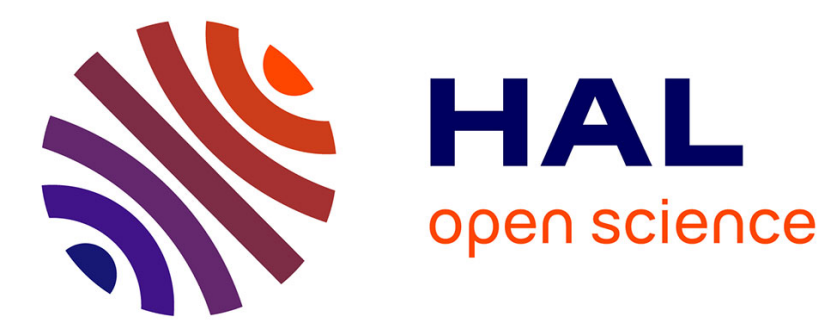

\title{
Lutte biologique contre la fusariose vasculaire de la tomate. Résultats en serre de production
}

Yvonne Couteaudier, Michel Letard, Claude Alabouvette, Jean Louvet

\section{To cite this version:}

Yvonne Couteaudier, Michel Letard, Claude Alabouvette, Jean Louvet. Lutte biologique contre la fusariose vasculaire de la tomate. Résultats en serre de production. Agronomie, 1985, 5 (2), pp.151156. hal-00884744

\author{
HAL Id: hal-00884744 \\ https://hal.science/hal-00884744
}

Submitted on 1 Jan 1985

HAL is a multi-disciplinary open access archive for the deposit and dissemination of scientific research documents, whether they are published or not. The documents may come from teaching and research institutions in France or abroad, or from public or private research centers.
L'archive ouverte pluridisciplinaire $\mathbf{H A L}$, est destinée au dépôt et à la diffusion de documents scientifiques de niveau recherche, publiés ou non, émanant des établissements d'enseignement et de recherche français ou étrangers, des laboratoires publics ou privés. 


\title{
Lutte biologique contre la fusariose vasculaire de la tomate. Résultats en serre de production
}

\author{
Yvonne COUTEAUDIER, Michel LETARD (*), Claude ALABOUVETTE \& Jean LOUVET \\ I.N.R.A., Station de Recherches sur la Flore pathogène dans le Sol, 17, rue Sully, F 21034 Dijon Cedex \\ (*) C.T.I.F.L., Station Régionale d'Expérimentation sur les cultures légumières, Allée des Sapins, F 44470 \\ Carquefou
}

Les connaissances acquises au cours de l'étude de la résistance des sols de Châteaurenard ont permis d'élaborer en conditions expérimentales 2 méthodes de lutte biologique contre les fusarioses vasculaires. Après avoir obtenu de bons résultats en conditions expérimentales, l'efficacité de ces procédés a été testée dans une serre dont le sol est très infesté par Fusarium oxysporum f. sp. lycopersici.

La première méthode consiste à mélanger à un sol préalablement traité à la chaleur une terre résistante épurée des microorganismes pathogènes qu'elle peut contenir. Alors que le traitement à la chaleur du sol infesté est insuffisant à lui seul pour assurer la protection de la culture, l'apport de $8 \mathrm{p} .100$ en volume de terre résistante épurée a permis de protéger efficacement 5 cultures successives de tomates pendant 3 années (tabl. 1).

La seconde méthode consiste à utiliser des souches de Fusarium non pathogènes sélectionnées pour leur capacité à coloniser intensément un sol traité à la chaleur. L'incorporation à ce sol de telles souches multipliées dans un substrat tourbeux a permis de diminuer fortement la gravité de la maladie. Ces résultats conduisent à étudier la dynamique des populations introduites dans les sols et indiquent que l'exploitation des relations de compétition entre Fusarium pathogènes et Fusarium non pathogènes peut déboucher sur un moyen de lutte efficace contre les fusarioses vasculaires.

Mots clés additionnels : Désinfection de sol, résistance, Fusarium, populations, recolonisation dirigée, compétition. greenhouse.

\begin{abstract}
Studies of the Fusarium wilt suppressiveness of the soil from Chateaurenard indicated 2 different approaches for controlling Fusarium wilts by biological procedures. Having obtained satisfactory data under experimental conditions, these procedures were tested in a commercial greenhouse where the soil was heavily infested by Fusarium oxysporum f, sp. lycopersici.

The first method was based on the transfer of the suppressiveness from a pathogen-free suppressive soil to the infested soil previously steam-treated. The pathogen-free suppressive soil was obtained by incorporating into the steamed suppressive soil from Châteaurenard cultures of non-pathogenic fungi, especially Fusarium oxysporum and Fusarium solani previously isolated from the same soil. A few days before planting, this pathogen-free suppressive soil was introduced at a rate of $8 \%$ to a depth of $20 \mathrm{~cm}$ into the infested soil, after steam-treatment. This procedure gave good control of Fusarium wilt during 3 consecutive years of tomato crops by comparison with the non-treated and steam-treated controls where the disease was severe (table 1).

The second method was based on the introduction of non-pathogenic Fusarium selected in laboratory tests for their ability to colonize a steam-treated soil intensively. Strains of non-pathogenic Fusarium grown in a fermenter were introduced into a steamed peat where they were multiplied by glucose amendment. After steam-treatment of the infested soil, this peat was introduced at a rate of $1 \mathrm{v}$. peat $/ 10 \mathrm{v}$. soil. Disease incidence was considerably reduced at the end of the first year of cropping. These results indicate that competition between non-pathogenic and pathogenic Fusarium could be a mechanism for controlling Fusarium wilts. Fusarium wilts.
\end{abstract}

Additional key words : Soil steam-treatment, suppressiveness, Fusarium, populations, controlled recolonization, competition.

\section{INTRODUCTION}

Dans des articles précédents, nous avons démontré qu'il est possible, par mélanges, de transmettre efficacement la résistance des terres de Châteaurenard, naturellement résistantes aux fusarioses vasculaires, aussi bien à des terres préalablement traitées à la vapeur qu'à des substrats de culture hors-sol (LOUVET et al., 1976 ; AlabouvetTe et al., 1980 ; COUTEAUDier \& AlabouvetTe, 1981). Ces résultats ont été 
acquis en conditions expérimentales contrôlées en présence d'un inoculum introduit massivement et réparti de façon homogène dans l'ensemble du volume de terre ou de substrat. En revanche, les expérimentations rapportées dans cet article sont réalisées dans des conditions d'infestation naturelle dans une serre de production. Leur but est de tester la validité de deux méthodes de protection biologique des cultures commerciales de tomates contre la fusariose vasculaire.

La première consiste à s'affranchir de la terre résistante naturelle de Châteaurenard tout en conservant l'essentiel de sa microflore et de ses propriétés; en effet, l'emploi direct de la terre naturelle de Châteaurenard présente l'inconvénient majeur de transmettre des agents pathogènes d'origine tellurique, en particulier Pyrenochaeta lycopersici Gerlach, agent de la maladie des racines liégeuses. Il a donc fallu éliminer les microorganismes qui constituent un danger potentiel pour les cultures tout en conservant la résistance, de façon à obtenir une terre résistante « épurée » utilisable comme moyen de lutte biologique.

D'autre part, à la suite des travaux de RouXEL et al. (1979) démontrant le rôle prépondérant des Fusarium oxysporum (Schlecht.) Snyd. \& Hans. et Fusarium solani (Mart.) Snyd. \& Hans. clans les mécanismes de la résistance du sol de Châteaurenard, nous avons enregistré des résultats expérimentaux intéressants par introduction massive dans un sol désinfecté de souches saprophytes sélectionnées (ALABOUVETTE et al., 1984b). C'est pourquoi la seconde méthode est basée sur l'incorporation au sol maraîcher de Fusarium antagonistes, installés et multipliés dans une tourbe, milieu de transfert compatible avec les pratiques culturales.

Les résultats présentés ici ont été obtenus au cours de 3 années consécutives dans une serre de production du Centre Technique Interprofessionnel des Fruits et Légumes (CTIFL), à Carquefou (Loire-Atlantique).

\section{MATÉRIEL ET MÉTHODES}

\section{A. Préparation des substrats de transfert de la résistance}

\section{Terre résistante épurée}

Cette épuration, réalisée par traitement ménagé de la terre $\left(70^{\circ} \mathrm{C}, 30 \mathrm{mn}\right)$, permet la destruction des champignons. Par la suite, 5 souches de $F$. oxysporum, 5 souches de $F$. solani et 16 souches appartenant aux genres Aspergillus (Michel) Lk., Cephalosporium Corda, Mucor Micheli, Nigrospora Zimm., Penicillium Link, Rhizopus Ehrenb. et Trichoderma Pers., toutes isolées du sol résistant de Châteaurenard, sont utilisées pour assurer la recolonisation fongique de la terre traitée. En effet, des résultats publiés précédemment (RouXEL et al., 1979) montrent que la réintroduction de la majorité de la mycoflore autochtone rétablit la résistance. Après avoir vérifié par inoculation l'innocuité des souches de Fusarium vis-à-vis de la tomate, chaque isolat est mis en culture sur bouillon de pomme de terre glucosé pendant une semaine. Les cultures sont homogénéisées au mixer, filtrées et incorporées à la terre traitée à la chaleur à raison de 1 volume de suspension pour 10 volumes de terre.
Afin de privilégier l'installation dans la terre des populations fusariennes, les Fusarium sont incorporés $15 \mathrm{j}$ avant les autres champignons. Le mélange obtenu est cultivé (en melons) puis dilué dans la terre traitée à la chaleur ; ce procédé maintient la résistance acquise à un niveau élevé (ALABOUVETTE, 1983) et, répété à différentes reprises, permet d'obtenir un volume important $\left(3 \mathrm{~m}^{3}\right)$ dont une partie est utilisée pour l'essai présenté ici. La méthode d'estimation de la réceptivité des sols aux fusarioses vasculaires (ALABOUVETTE et al., 1982) permet de vérifier que le niveau de résistance obtenu est toujours comparable à celui du sol résistant d'origine.

\section{Tourbe enrichie en Fusarium antagonistes}

Deux souches de $F$. oxysporum et deux souches de $F$. solani, sélectionnées pour leur aptitude à se multiplier intensément dans un substrat désinfecté et à y exprimer des capacités antagonistes, ont été mises en culture pendant $10 \mathrm{j}$ sur bouillon de pomme de terre glucosé. A l'issue de cette période, chaque culture a été centrifugée ( 3500 tours, $20 \mathrm{mn}$ ) puis mise en suspension dans de l'eau stérile avant d'être incorporée dans du talc stérile à raison de $1 \mathrm{ml}$ pour $2 \mathrm{~g}$ de talc. Après séchage à $20^{\circ} \mathrm{C}$ et stockage en récipient clos, on peut conserver pendant plusieurs mois cet inoculum concentré ( $10^{6}$ à $10^{7}$ propagules $/ g$ ), (TELlo MARQUINA \& AlabouvetTE, 1984). Une tourbe blonde finlandaise fertilisée, de type commercial, traitée pendant $1 \mathrm{~h}$ à la vapeur à $100^{\circ} \mathrm{C}$, est divisée en 4 lots. Chaque souche est introduite dans un lot à raison de $10^{5}$ propagules $/ \mathrm{ml}$; les 4 lots sont mis en incubation à $25^{\circ} \mathrm{C}$ et homogénéisés régulièrement. Quinze jours avant la mise en place de l'essai, ils sont mélangés puis amendés avec du glucose à la dose de $1 \mathrm{mg} / \mathrm{ml} \mathrm{de}$ façon à permettre la multiplication des souches dans leur substrat de transfert. Une analyse de cette tourbe enrichie en Fusarium saprophytes, par la technique des suspensions-dilutions, est réalisée au moment de son incorporation au sol ; le niveau des populations de Fusarium s'établissait alors à $3.10^{5}$ propagules $/ \mathrm{ml}$ de tourbe.

\section{B. Protocole expérimental}

Le sol de la serre dans laquelle sont réalisées les expérimentations à partir de 1981 est gravement infesté depuis plusieurs années par $F . O$. f. sp. lycopersici. Il est divisé en 3 parcelles (A, B, C) de $25 \mathrm{~m}^{2}$ chacune qui sont traitées comme suit :

- en 1981 : traitement à la vapeur $(12 \mathrm{~h}$ sous bâche) des parcelles $\mathrm{B}$ et $\mathrm{C}$; puis $3 \mathrm{j}$ après désinfection, incorporation de 8 p. 100 en volume de terre résistante épurée à la parcelle $C$ sur $20 \mathrm{~cm}$ de profondeur, A étant conservée comme témoin ;

- en 1983 : traitement à la vapeur de la parcelle D (anciennement parcelle témoin A), puis incorporation sur $20 \mathrm{~cm}$ de tourbe enrichie en Fusarium saprophytes (1 v./10 v.).

La serre est exploitée en monoculture, 64 plants de tomate préparés en terreau désinfecté sont repiqués dans chaque parcelle au printemps et à l'automne. La maladie ne se manifestant pas ou peu au cours des cultures de printemps, les notations de gravité de la 
maladie sont faites sur les cultures d'automne 4 mois et demi après plantation des variétés «Vémone 》 en 1981, « Lucy » en 1982 et 1983.

\section{Notation des symptômes}

A la fin de chaque culture, le taux de plantes infectées est estimé par l'examen du brunissement vasculaire et confirmé par des isolements microbiologiques. Un indice de flétrissement basé sur les symptômes foliaires observés sur chaque plante donne une évaluation du degré de flétrissement des plantes :

0 - pas de symptôme

1 - jaunissement d'une foliole ou d'une feuille

2 - jaunissement sur 1 à 2 feuilles

3 - jaunissement sur plus de 2 feuilles

4 - jaunissement et dessèchement de la majorité des feuilles

5 - plante morte.

\section{RÉSULTATS}

\section{A. Persistance de la protection biologique obtenue par utilisation de terre résistante}

Les taux élevés de plantes infectées enregistrés en 1981 et 1982 dans les parcelles A et B (tabl. 1) traduisent une infestation très importante du sol cultivé.

Le traitement à la vapeur de la parcelle B en 1981

TABLEAU 1

Gravité de la fusariose vasculaire de la tomate après différents traitements $d u$ sol

dans une serre naturellement infestée par $\mathrm{F}$. o. $f . s p$. lycopersici.

$A$ : parcelle témoin.

$B$ : parcelle traitée 12 h à la vapeur en juillet 1981.

$C$ : parcelle traitée $12 \mathrm{~h}$ à la vapeur en juillet 1981 puis apport de terre résistante épurée avant culture $(8 \mathrm{p} .100$ en volume $s u r 20 \mathrm{~cm}$ de profondeur).

$D$ : parcelle traitée à la vapeur en 1983 puis apport de Fusarium antagonistes (ancienne parcelle A).

64 plantes/parcelle $\left(25 \mathrm{~m}^{2}\right)$.

Incidence of Fusarium wilt of tomatoes after different soil treatments in a greenhouse soil naturally infested by $\mathrm{F}$. o. $f$. sp. lycopersici.

$A:$ non-treated plot.

$B$ : plot steam-treated for $12 \mathrm{~h}$ in July 1981

$C$ : plot steam-treated as plot $B$ and then amended with a pathogen-free suppressive soil at a rate of $8 \%$ to a depth of $20 \mathrm{~cm}$.

$D:$ plot steam-treated before introduction of non-pathogenic Fusarium (1983).

64 plants/plot $\left(25 \mathrm{~m}^{2}\right)$

\begin{tabular}{ccccccc}
\hline \multicolumn{7}{c}{ Gravité de la maladie } \\
\hline Parcelles & \multicolumn{7}{c}{ (a) $^{1981}$} & (b) & (a) & (b) & (a) & (b) \\
\hline A & 42 & 31 & 83 & 54 & & - \\
\hline B & 55 & 48 & 89 & 72 & 98 & 91 \\
\hline C & 6 & 2 & 11 & 4 & 25 & 17 \\
\hline D & & & & & 20 & 15 \\
\hline
\end{tabular}

(a) Pourcentages de plantes infectées ; (b) indice de flétrissement évalué sur une échelle de 0 à 5,4 mois et demi après plantation.

(a) Percentages of diseased plants; (b) disease severity evaluated on a scale of $0-5,4.5$ months after plantation. s'est avéré inefficace pour désinfecter le sol. Le niveau de maladie est même constamment plus élevé dans la parcelle ainsi traitée que dans le témoin (parcelle A) ; il semble que l'inoculum résiduel soit favorisé par rapport aux autres microorganismes dans la recolonisation du sol.

Par contre, le faible niveau de maladie enregistré dans la parcelle $\mathrm{C}$ indique clairement que l'apport de terre résistante épurée confère au sol une bonne résistance à la maladie: dès 1981 , la gravité de la maladie a diminué proportionnellement de 86 p. 100 par rapport à la parcelle témoin $\mathrm{A}$ non traitée. En outre, ce niveau de résistance acquise s'est maintenu au cours des cultures successives : en 1982 , après la $3^{\mathrm{e}}$ culture, la diminution proportionnelle de la gravité de la maladie atteint 87 p. 100 ; en 1983 , le taux de plantes infectées à l'issue de la $5^{\mathrm{e}}$ culture montre que le niveau de maladie demeure faible.

\section{B. Lutte biologique par utilisation de microorga- nismes antagonistes}

L'apport de tourbe enrichie en Fusarium saprophytes dans le sol traité à la vapeur (tabl. 1) permet une réduction très nette de la gravité de la maladie puisqu'en fin de culture le pourcentage de plantes parasitées est de 98 p. 100 dans la parcelle B et de 20 p. 100 dans la parcelle $\mathrm{D}$ amendée avec des microorganismes antagonistes; les indices de flétrissement correspondants traduisent le gain de production apporté par ce traitement biologique.

\section{CONCLUSION ET DISCUSSION}

Les niveaux élevés de protection obtenus dans les conditions pratiques de culture en sol naturellement très infesté apportent la preuve qu'il est possible de limiter efficacement une fusariose vasculaire en modifiant l'activité microbienne d'un sol.

Le traitement du sol à la vapeur est totalement inefficace à lui seul pour lutter contre la maladie ; nous nous trouvons sans doute dans les conditions décrites par MAROIS et al. (1983) qui démontrent que la fumigation des sols est suivie d'une recolonisation très rapide par les $F$. oxysporum pathogènes hébergés dans les zones du sol non atteintes par le traitement. Par contre, si la perturbation microbiologique créée par la désinfection est suivie d'une recolonisation dirigée, obtenue par l'incorporation au sol soit de terre résistante épurée, soit de microorganismes antagonistes, elle s'accompagne d'un effet positif extrêmement net sur l'état sanitaire de la culture; en effet, celui-ci s'avère encore satisfaisant 3 ans après le traitement du sol par de la terre résistante, ce qui indique la persistance de la résistance transmise. Peu de travaux concernant la transmission de la résistance des sols aux fusarioses vasculaires décrivent un effet durable en conditions d'infestation naturelle. Si SCHER \& BAKER (1980) luttent efficacement 2 années de suite contre la fusariose de l'œillet par utilisation de faibles quantités de sol californien, TU \& CHENG (1981), protégeant une culture de bananier par incorporation de sol résistant de Changwa dans les trous de plantation, observent une recrudescence de la maladie dès la $2^{\mathrm{e}}$ année de culture. 
On doit s'interroger sur les mécanismes microbiologiques impliqués dans le processus de transmission de la résistance. En ce qui concerne les sols californiens, SCHER \& BAKER (1982) attribuent un rôle prépondérant aux Pseudomonas fluorescents produisant des sidérophores capables de complexer le fer nécessaire au développement des Fusarium. Dans le cas présent, cette hypothèse n'a pas été prise en considération car la terre résistante épurée ayant été préalablement traitée à la chaleur, il est permis de penser que les bactéries thermosensibles du type Pseudomonas ont été éliminées. Cependant, l'apport de terre résistante épurée ne se limite pas à un simple apport de Fusarium saprophytes, l'environnement constitué par la terre de Châteaurenard intervenant sans doute dans l'expression de la résistance obtenue (RouXEL et al., 1979).

D'autre part, si le sol de Châteaurenard se révèle un substrat intéressant pour accueillir et transférer la résistance, un tel sol ne peut être à l'origine d'un procédé de lutte fiable et économiquement utilisable dans les conditions de la production. C'est pourquoi nous avons eu recours à des Fusarium saprophytes sélectionnés qui se révèlent être des microorganismes antagonistes utilisables en lutte biologique. Dès 1946, Van KoOT mentionne l'introduction de $F$. solani dans un sol traité à la chaleur pour lutter contre la fusariose vasculaire du concombre. TRAMIER et al. (1983) et GARIBALDI (1984) tentent d'utiliser ce type d'intervention pour lutter contre la fusariose vasculaire de l'œillet ; toutefois, ils ne donnent pas d'informations sur le choix des différentes souches de champignons et sur le mode d'inhibition du pathogène. Les bons résultats enregistrés ici, en serre naturellement infestée, indiquent que le potentiel infectieux du sol de la parcelle D traitée avec les souches saprophytes a diminué, ce qui s'est traduit par une faible gravité de la maladie. Des analyses microbiologiques réalisées en fin de culture montrent que l'agent pathogène a fortement recolonisé le sol de la parcelle $\mathrm{B}$ à la suite du traitement à la vapeur effectué en 1981. Au contraire, dans la parcelle $\mathrm{D}$, la flore fusarienne introduite avec le substrat tourbeux enrichi s'est bien installée dans le sol où elle a limité la recolonisation par l'agent pathogène. Ces résultats tentent à conforter l'hypothèse selon laquelle une compétition intragénérique, liée à l'état nutritif du sol (ALABOUvETTE et al., 1984a) s'exprime dans un sens d'autant plus défavorable à l'expression des $F$. oxysporum pathogènes qu'ils sont en plus faible proportion parmi la flore fusarienne du sol. Cela incite à approfondir l'étude du mécanisme de compétition intragénérique afin de préciser l'importance du rapport :

densité de la population de F. oxysporum pathogène densité de la population fusarienne totale

ROUXEL et al. (1979) ont montré qu'un apport de $F$. oxysporum et de $F$. solani suffisait à rétablir la résistance dans le sol de Châteaurenard traité à la chaleur ; mais ils ont échoué en essayant d'établir une résistance dans un sol sensible particulier par introduction de ces mêmes souches. Cela ne signifie pas pour autant qu'aucune terre sensible ne soit apte à permettre l'installation et l'expression des capacités antagonistes de Fusarium spp. exogènes préalablement sélectionnés.

Un test mis au point au laboratoire nous permet dès maintenant de sélectionner les souches pour leur aptitude à se multiplier intensément dans un sol désinfecté; des résultats préliminaires indiquent que la dynamique de colonisation par une population donnée de Fusarium varie selon les caractéristiques du milieu colonisé. Simultanément, notre attention se porte sur l'aptitude des différentes souches sélectionnées à inhiber l'expression des capacités infectieuses de l'agent pathogène.

Un procédé de lutte biologique efficace, basé sur l'utilisation de Fusarium saprophytes, champignons ubiquistes faciles à multiplier en masse et à conserver avant leur utilisation, devra également tenir compte des garanties d'innocuité et de stabilité des souches. Néanmoins, avec SCHROTH \& HANCOCK (1982), nous pensons que l'incorporation au sol de microorganismes antagonistes adaptés à cet environnement devrait aboutir à une protection durable, ce qui devra être vérifié en poursuivant les observations phytosanitaires sur cet essai.

Recu le 13 mai 1984. Acceplé le 2 ocrobre 1984.

\section{RÉFÉRENCES BIBLIOGRAPHIQUES}

Alabouvette C., 1983. La réceptivité des sols cux fusarioses vasculaires. Rôle de la compétition nutritive entre microorganismes. Thèse Doct. ès-Sci. Nat., Univ. de Nancy, 158 p.

Alabouvette C., Couteaudier Y., Louvet J., 1982. Comparaison de la réceptivité de différents sols et substrats de culture aux fusarioses vasculaires. Agronomie, 2 (1), 1-6.

Alabouvette C., Couteaudier Y., Louvet J., 1984a. L'antagonisme microbien : importance des phénomènes de compétition. $24^{\mathrm{C}}$ Coll. Soc. Fr. Phytopathol., Agronomie, 4 (2), 200.

Alabouvette C., Couteaudier Y., Louvet J., 1984b. Fusarium wiltsuppressive soils : mechanisms of suppression and management of suppressiveness. In C. A. Parker, K. J. Moore, P. I. W. Wong, A. D. Rovira and J. F. Kollmorgen : Ecology and Management of Soil Borne Plant Pathogens. Am. Phytopathol. Soc., St Paul MN, (sous presse).
Alabouvette C., Tramier R., Grouet D., 1980. Recherches sur la résistance des sols aux maladies. VIII. Perspectives d'utilisation de la résistance des sols pour lutter contre les fusarioses. Ann. Phytopathol., 12 (2), 83-93.

Couteaudier Y., Alabouvette C., 1981. Fusarium wilt diseases in soilless cultures. Acta Hortic., Substrates in Hortic., 126, 153-157.

Garibaldi A.. 1985. Researches in Italy on soils suppressive to Fusarium wilt of carnation. Phytopathol. mediterr., (sous presse).

Komada H., 1975. Development of a selective medium for quantitative isolation of Fusarium oxysporum from natural soil. Rev. Plant Prot. Res., Tokyo, 8, 114-125.

Louvet J., Rouxel F., Alabouvette C., 1976. Recherches sur la résistance des sols aux maladies. I. Mise en évidence de la nature microbiologique de la résistance d'un sol au développement de la fusariose vasculaire du melon. Ann. Phytopathol., 8, 425-436. 
Marois J. J., Dunn M. T., Papavizas G. C., 1983. Reinvasion of fumigated soil by Fusarium oxysporum f. sp. melonis. Phytopathology, 73 (5), 680-684.

Rouxel F., Alabouvette C., Louvet J., 1979. Recherches sur la résistance des sols aux maladies, IV. Mise en évidence du rôle des Fusa rium autochtones dans la résistance d'un sol à la fusariose vasculaire du melon. Ann. Phytopathol., 11, 199-207.

Rouxel F., Bouhot D., 1971. Recherches sur l'écologie des champignons parasites dans le sol. IV. Nouvelles mises au point concernant l'analyse sélective et quantitative des Fusarium oxysporum et Fusarium solani dans le sol. Ann. Phytopathol., 3, 171-183.

Scher M. F., Baker R., 1980. Mechanism of biological control in Fusarium-suppressive soil. Phytopathology, 70, 412-417.

Scher M. F., Baker R., 1982. Effect of Pseudomonas putida and a synthetic iron chelator on induction of soil suppressiveness to Fusarium wilt pathogens. Phytopathology, 72, 1567-1573.
Schroth M. N., Hancock J. G., 1982. Disease-suppressive soil and root-colonizing bacteria. Science, 216 (25), 1376-1381.

Tello-Marquina J. C., Alabouvette C., 1984. Observations sur la persistance dans le sol des microconidies de Fusarium oxysporum. Agronomie, 4 (9), 885-890.

Tramier R., Pionnat J. C., Tebibel N., 1983. Role of the fungi in the induction of suppressiveness into substrates to Fusarium wilt of carnation. Acta Hortic., 141, 55-59.

Tu C. C., Cheng Y. H., 1981. Soil microbial activity in relation to Fusarium wilt-suppressive soil and conductive soil. Taïwan DAIS Sci. Meeting Report, 1-12.

Van Koot Y., 1944. De Fusariumziekte van komkommer en meloen. Meded. Tuinbouwvoorlichtingsdienst. 42, $85 \mathrm{p}$. 